https://helda.helsinki.fi

Long-Term Dynamics in a Metapopulation of the American Pika

\title{
Moilanen, Atte
}

1998

pÿThe American Naturalist. 152(4): 530542.1998

http://hdl.handle.net/1975/7568

http://dx.doi.org/10.1086/286188

Downloaded from Helda, University of Helsinki institutional repository.

This is an electronic reprint of the original article.

This reprint may differ from the original in pagination and typographic detail.

Please cite the original version. 


\title{
Long-Term Dynamics in a Metapopulation of the American Pika
}

\author{
Atte Moilanen, ${ }^{1, *}$ Andrew T. Smith, ${ }^{2}$ and Ilkka Hanski ${ }^{1}$
}

1. Department of Ecology and Systematics, Division of Population Biology, P.O. Box 17 (Arkadiankatu 7), FIN-00014 University of Helsinki, Finland;

2. Department of Zoology, Box 871501, Arizona State University, Tempe, Arizona 85287-1501

Submitted March 19, 1997; Accepted April 1, 1998

ABSTRACT: A 20-yr study of a metapopulation of the American pika revealed a regional decline in occupancy in one part of a large network of habitat patches. We analyze the possible causes of this decline using a spatially realistic metapopulation model, the incidence function model. The pika metapopulation is the best-known mammalian example of a classical metapopulation with significant population turnover, and it satisfies closely the assumptions of the incidence function model, which was parameterized with data on patch occupancy. The model-predicted incidences of patch occupancy are consistent with observed incidences, and the model predicts well the observed turnover rate between four metapopulation censuses. According to model predictions, the part of the metapopulation where the decline has been observed is relatively unstable and prone to large oscillations in patch occupancy, whereas the other part of the metapopulation is predicted to be persistent. These results demonstrate how extinction-colonization dynamics may produce spatially correlated patterns of patch occupancy without any spatially correlated processes in local dynamics or extinction rate. The unstable part of the metapopulation gives an empirical example of multiple quasi equilibria in metapopulation dynamics. Phenomena similar to those observed here may cause fluctuations in species' range limits.

Keywords: metapopulation, incidence function, Ochotona princeps, regional stochasticity, multiple equilibria, stepping-stone.

Distribution and abundance of species do not typically remain constant for long periods of time (Taylor and Woiwod 1980; Pimm 1991; Gaston 1994; Brown 1995). Much of population ecology consists of studies of changing distribution and abundance and of the processes that

* To whom correspondence should be addressed; E-mail: Atte.Moilanen@ Helsinki.Fi.

Am. Nat. 1998 . Vol. 152 , pp. $530-542$. (c) 1998 by The University of Chicago. 0003-0147/98/5204-0003\$03.00. All rights reserved. cause these changes. There are good theoretical and empirical reasons to assume that many long-term changes in the distribution and abundarice of species reflect longterm environmental changes, but one should not ignore alternative explanations. For instance, recent theoretical studies have shown that the dynamics of spatially extended populations may show complex transient behavior (Hastings and Higgins 1994; White and Bowers 1996) and alternative stable equilibria (Gyllenberg and Hanski 1992). An empirical example of the latter can be found elsewhere (Hanski et al. 1995b). Dramatic changes in the distribution and abundance of species may thus occur in the absence of any long-term environmental trends.

A long-term study of a metapopulation of the American pika (Ochotona princeps) in California has revealed a regional decline in habitat occupancy over a period of about 20 yr (Smith 1974a, 1980; Smith and Gilpin 1997). In the southern part of the study area, patch occupancy declined from $54 \%$ in 1972 to $4 \%$ in 1991, whereas, in the northern part, patch occupancy remained at a relatively constant and high level (fig. 1). The observed regional decline in patch occupancy is puzzling because the habitat and climate are uniform across the small study area, and no changes in the environment quality have been observed during the 20-yr study period (Smith and Gilpin 1997). In this study, we examine the long-term behavior of the pika metapopulation from the viewpoint of classical metapopulation dyaamics. Specifically, we attempt to assess whether the observed pattern of change in the distribution of the pika is consistent with metapopulation dynamics in a constant environment.

In this study, we apply a spatially realistic metapopulation model, the incidence function model (Hanski 1994), which has been used previously in studies of butterfly metapopulations (Hanski et al. 1996a, 1996b; Wahlberg et al. 1996). The incidence function model has several features that make it a realistic description of many metapopulations: the spatial structure of the habitat is modeled explicitly, colonization of empty patches is distance dependent, large patches send out more migrants than small ones, the extinction probability of a local population depends on population size (via patch size), and the 


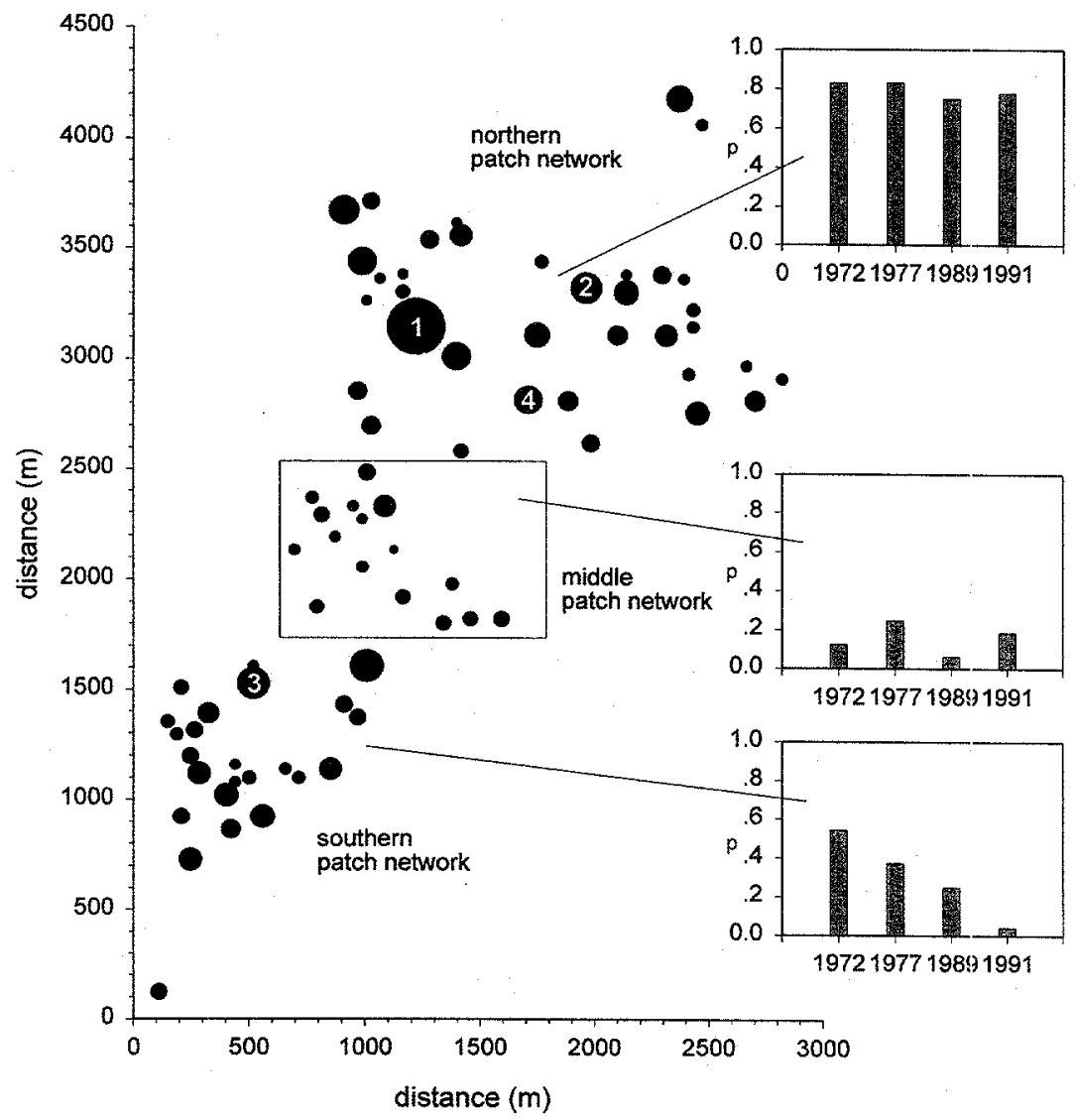

Figure 1: The habitat patch structure of the Bodie pika metapopulation. Circle area is proportional to the estimated carrying capacity of the patch. The figure shows the division of the patches into northern, middle, and southern patch networks, including 36 , 16 , and 24 patches, respectively. The small panels show the observed patch occupancies ( $p=$ proportion of patches occupied) in the respective parts of the metapopulation in the four surveys. In the north, the level of patch occupancy has varied little during the $20 \mathrm{yr}$, whereas there has been a distinct decline in occupancy in the southern part of the metapopulation. Patches $1-4$ are the four patches having the greatest value as measured by our measure of colonization potential. Effects of removing these patches are examined in figure 8 .

effect of immigration on extinction risk is included in the model. The incidence function model can be parameterized with a snapshot of patch occupancy data (Hanski 1994; ter Braak et al. 1998), and it can be used to make quantitative predictions about metapopulation dynamics in specific patch networks (Wahlberg et al. 1996). Here, we first parameterize the incidence function model for the American pika, then investigate the dynamics of the metapopulation with the parameterized model, both with and without additional regional stochasticity.

\section{Material and Methods}

\section{The Bodie Pika Metapopulation}

The pika metapopulation is located in the largely abandoned mining area of Bodie, Mono County, California. It has been the subject of a long-term research project (Smith 1974a, 1974b, 1978, 1980; Smith and Gilpin
1997). The Bodie pika metapopulation fulfills exceptionally well the four conditions of regional persistence of a species as a classical metapopulation (Hanski et al. 1995a). First, the pika lives in spatially distinct habitat patches-piles of broken rock adjoining vegetation suitable for foraging. At Bodie, this type of habitat (mine tailings) is sparsely scattered across an open landscape of sagebrush, which is not suitable for the pika (Smith $1974 a$ ). Second, there is no "mainland" population, and, thus, all local populations have a significant risk of extinction. At Bodie, the estimated size of the largest local population is circa 50 individuals, which is hardly enough to make it entirely safe from extinction. The median population size in 1972 was only three individuals. Third, migration is distance dependent, and, hence, the spatial configuration of the habitat has significant consequences for the dynamics, even though the habitat patch network covers only an area of circa $12 \mathrm{~km}^{2}$ (fig. 1). This 
pika metapopulation is located close to the range limit of the species, and movements of pikas outside habitat patches are restricted by high daytime temperatures at Bodie (Smith 1974a, 1974b). In a recent study (Peacock 1995; Peacock and Smith 1997), only one out of 105 marked adult pikas moved a short distance to a neighboring patch. In the same study, 11 juvenile individuals were observed to migrate between patches, and the average migration distance was only $133 \mathrm{~m}$. In another study, John Nagy (personal communication) marked juvenile pikas and followed them for 1 yr. Five out of 34 marked individuals survived, and only two of them migrated to another patch (distances 60 and $150 \mathrm{~m}$ ). In summary, these results indicate a low emigration rate, especially in adult pikas, and short migration distances. Fourth, the dynamics of local populations appear to be sufficiently asynchronous (Smith 1980; Smith and Gilpin 1997) to make the metapopulation relatively safe from simultaneous extinction of all local populations. We conclude that the Bodie pika metapopulation is the best mammalian example known to us of a classical metapopulation with significant population turnover.

Pikas have been known to inhabit the Bodie region for a long time, probably since before the turn of the century (Smith and Gilpin 1997). The spatial configuration of the mine tailing patches has remained essentially unchanged since the 1940s, and there have been no apparent environmental changes in the area during the last $50 \mathrm{yr}$ (Severaid 1955; Smith and Gilpin 1997; A. T. Smith, personal observation). Thus there are no reasons to assume that the Bodie pika metapopulation would be tracking a changing environment. Detailed population studies of the pika were initiated in 1969 , and essentially the entire metapopulation was censused in 1972, 1977, 1989, and 1991. Pikas are individually territorial animals, and their characteristic vocalizations and feces, as well as the conspicuous hay piles that they gather for winter, can be detected easily (Smith 1974a). We are therefore confident that the censuses have produced accurate data on the presence of pikas in the set of habitat patches. The data set analyzed in this study includes 76 habitat patches. We have omitted four very small patches that were not included in all censuses. Unfortunately, a few mediumsized patches located near the two northernmost patches in figure 1 could not be censused due to lack of access to these patches on private land (Smith and Gilpin 1997).

\section{The Incidence Function Model}

The incidence function model (Hanski 1994) is based on a first-order, linear Markov chain model for the occupancy of a single habitat patch. A habitat patch has two possible states, occupied or empty. Changes in the state of patch $i$ are determined by extinction and colonization probabilities $E_{i}$ and $C_{i}$, which are calculated independently for each patch $i$ in each time unit. The model has six parameters, $x, y, e, \alpha, A_{0}$, and $b$ as described below, and it uses patch area $A_{i}$ and patch connectivity $S_{i}$ (originally referred to as "isolation" in Hanski 1994) as variables.

The extinction probability is given by a power function of patch area, $E_{i}=e / A_{i}^{x}$, on the assumptions that the expected population size is positively correlated with patch size (as observed for the pikas; Smith 1974a) and that small populations are more likely to go extinct than large ones (for the pikas, see Smith 1980). The power function relationship between $E_{i}$ and $A_{i}$ can be justified on the basis of a standard extinction model (Hanski 1998). For simplicity, connectivity is not assumed to affect the intrinsic risk of extinction (but see the rescue effect below) The colonization probability of patch $i$ is defined by a sigmoid function $C_{i}=S_{i}^{2} /\left(S_{i}^{2}+y^{2}\right)$, where the connectivity $S_{i}$ of patch $i$ is defined by

$$
S_{i}=\sum_{j \neq i} p_{j} \exp \left(-\alpha d_{i j}\right) A_{j}^{b},
$$

where $p_{j}$ is the observed incidence for the patch, $d_{i j}$ is the distance between patches $i$ and $j$, and $\alpha$ is a constant setting the distance dependence of migration rate. If only one snapshot of data is available, then $p_{j}$ equals 1 for occupied and 0 for empty patches, but if several snapshots are available, one can use the observed average patch occupancy as $p_{j}$. Because the sizes of the source populations evidently affect the rate of migration to the focal patch $i$, $A_{j}$ is included in equation (1). The exponent $b$ scales the expected population size to patch area; it is best estimated from empirical data on population sizes.

Assuming a quasi-stationary state of patch occupancy, the model parameters $x, y$, and $e$ can be estimated from one or more snapshots of patch occupancy patterns by maximum likelihood regression between the empirically observed patch occupancies $p_{i}$ and the predicted longterm probability of patch occupancy (incidence) $J_{i}$ (Hanski 1994):

$$
\begin{aligned}
& \text { minimize } \\
& \theta=\sum_{\text {all } i}-p_{i} \log \left(J_{i}\right)-\left(1-p_{i}\right) \log \left(1-J_{i}\right),
\end{aligned}
$$

where the expression for $J_{i}$ is derived from the two-state Markov chain as

$$
J_{i}=\frac{C_{i}}{C_{i}+E_{i}}=\left\{1+\left[1+\left(\frac{y}{S_{i}}\right)^{2}\right] \frac{e}{A_{i}^{x}}\right\}^{-1} .
$$

It is often appropriate to use a model variant that includes the rescue effect, a lowered risk of extinction of lo- 
cal populations due to immigration increasing local population size (Brown and Kodric-Brown 1977). Of course, emigration increases extinction rate in the source populations, but most migrants are produced by large populations; the negative impact of emigration on large populations is smaller than the positive impact of immigration to small populations (Hanski 1997). A simple approach to including the rescue effect into the incidence function model is to replace $E_{i}$ by $\left(1-C_{i}\right) E_{i}$, which yields a modified equation for $J_{i}$ :

$$
J_{i}=\frac{C_{i}}{C_{i}+E_{i}-E_{i} C_{i}}=\left(1+\frac{e^{\prime}}{S_{i}^{2} A_{i}^{x}}\right)^{-1},
$$

where $e^{\prime}=e y^{2}$. Note that replacing $E_{\mathrm{i}}$ by $\left(1-C_{i}\right) E_{i}$ does not assume simultaneous extinctions and recolonizations in the same time interval; rather, this is an attempt to model a reduction in extinction rate due to past immigration (see Hanski 1997; ter Braak et al. 1998). Equation (3) assumes that $C_{i}$ is constant, which can be only approximately correct since the $C_{i}$ values change from year to year as the pattern of patch occupancy changes. The $J$ values, not assuming constant $C$ values, can be obtained from long simulation runs of the Markov chain model (for particular parameter values) as the fraction of time each patch was occupied. This method was used to calculate $J$ values for figure $5 \mathrm{~A}$.

The version of the incidence function model with the rescue effect (eq. [3b]) is generally preferable (Hanski 1994). When using equation (3b), some additional information is needed to obtain independent estimates of the values of $e$ and $y$ in the product $e^{\prime}$. This can be achieved by estimating a minimum patch area $A_{0}$, for which the yearly extinction probability equals unity, $E_{0}=e / A_{0}^{x}=1$. It then follows that $e=A_{0}^{x}$ and $y=\sqrt{\left(e^{\prime} / e\right)}$. One can also use information about population turnover events between two snapshots to separate $e$ and $y$ from $e^{\prime}$ (for details, see Hanski 1994, 1996; ter Braak et al. 1998). One should also note that for the purpose of predicting the stochastic equilibrium ( $J$ values) with the estimated parameter values, a range of reasonable $e$ and $y$ values with a constant product $\left(e^{\prime}\right)$ will give essentially the same answer; only the predicted turnover rate is critically affected by the values of $e$ and $y$. A more technical assessment of the incidence function model is presented by ter Braak et al. (1998). Other commonly used metapopulation models can be derived as special cases of the incidence function model. The incidence function model collapses back to the Levins model (Levins 1969) when there is no variation in patch sizes and colonization is not affected by distance $(\alpha=0)$. A mainland-island situation (Harrison $1991,1994)$ can be modeled by including a large mainland patch, with a low extinction probability, into the patch network.
To iterate the dynamics of a metapopulation with the incidence function model, one first calculates the yearly colonization probability $C_{i}$ for each patch based on the patch occupancy pattern of the previous year. Next, the extinction probability in the presence of the rescue effect is calculated as $\left(1-C_{i}\right) e / A_{i}^{x}$ for each occupied patch. The actual extinctions and colonizations are then determined by comparing random numbers with the calculated extinction and colonization probabilities. Iterating metapopulation dynamics in this manner leads to stochastic variation in the number of occupied habitat patches (extinction-colonization stochasticity).

Another type of stochasticity that is likely to affect most natural metapopulations is called "regional stochasticity" (Hanski 1991) and is defined as spatially correlated environmental stochasticity. In this study, regional stochasticity was added to the model by multiplying patch areas in each year with a $\left(0, \sigma^{2}\right) \log _{10}$ normally distributed variable and variance $\sigma^{2}$ truncated upward at 1 . This distribution is justified for the pika as follows. The number of territories available for the pika does not change between years and rnost available territories tend to be occupied (Smith 1974a). Therefore few new territories can become available during "good" years, and it is reasonable to adjust effective patch areas only downward, which reflects extra spatially correlated mortality in "bad" years. Thus, on average, half of the years will have the multiplier for patch areas equaling 1 , half of the years will have a multiplier $<1$. It is reasonable to assume that regional stochasticity is synchronous across the entire metapopulation because the geographic area covered by the Bodie pika metapopulation is small and no differences in patch quality or weather conditions have been observed in any subregion of the patch network.

When studying the population dynamic consequences of a change in the spatial structure of a patch network, one is interested in the roles that individual patches play in the dynamics of the metapopulation. Here we suggest a simple measure that can be used in this context to assess the importance of particular patches to the dynamics of the metapopulation. We call this measure the colonization potential of patch $i, O_{i}$, and it is defined as the expected pooled population size in other patches that the focal population $i$ may colonize in one time unit:

$$
\begin{aligned}
O_{i}= & \sum_{j \neq i} P(\text { patch } i \text { colonizes patch } j) \\
& * \text { (expected population size of patch } j) \\
= & \sum_{j \neq i} J_{i} \frac{S_{i j}^{2}}{S_{i j}^{2} y^{2}} A_{j}^{b}:=\sum_{j \neq i} J_{i} \frac{e^{-\alpha d_{i j}} A_{i}^{b}}{e^{-\alpha d_{i}} A_{i}^{b}+y^{2}} A_{j}^{b},
\end{aligned}
$$

where $S_{i j}$ is the contribution of patch $i$ to the connectivity 
of patch $j$ (see eq. [1]) and $J_{i}$ is the incidence of patch $i$ (eq. [3b]). The colonization potential takes into account the frequency of, occupancy of, and number of migrants that patch $i$ produces $\left(J_{i} A_{i}^{b}\right)$, as well as the distances to $\left[\exp \left(-\alpha d_{i j}\right)\right]$ and the sizes of the target patches $\left(A_{j}^{b}\right)$. A patch with a large colonization potential has a large area, which will cause it to be occupied relatively often and to send out many migrants when occupied. However, even a large patch in the middle of nowhere will not play a significant part in the dynamics of any metapopulation. An "important" patch should, therefore, be centrally located since migrants moving out from a central location will easily find other patches and a centrally located population is likely to become quickly recolonized following an extinction. In a central location, the positive feedback caused by the rescue effect is also beneficial for the population. Finally, colonization of large patches will benefit the metapopulation more than colonization of small patches, hence the final term $\left(A_{j}^{b}\right)$ in equation (4).

\section{Results}

\section{Parameter Estimation}

The incidence function model has six parameters. Three parameters were estimated with independent biological data, while the remaining three parameters were estimated from the spatial pattern of patch occupancy. The exponent $b$ in equation (1) scales the local population size to patch area. In this study, patch "area" is measured by the length of patch perimeter because the pikas only use the margins of the mine tailings (Smith 1974a). The value of $b$ was obtained by regressing the observed population sizes against patch area. Using pooled data for all extant pika populations in the four surveys, we obtained an estimate of $b=0.74$.

The value of $A_{0}$ gives the size of a patch for which the yearly extinction probability equals unity. This value is used to tease apart the values of $e$ and $y$ in the product $e^{\prime}=e y^{2}$ (see model description, eq. [3b] and below). The smallest area that has been observed to show signs of pika occupation at Bodie is $10 \mathrm{~m}$ (Smith 1974a), and a few patches with the size of $15 \mathrm{~m}$ have been observed to be occupied several times. We selected $15 \mathrm{~m}$ for $A_{0}$, since some patches with area $<A_{0}$ are expected to be occupied in a large network due to colonization in the previous time unit (ter Braak et al. 1998).

Parameter $\alpha$ in equation (1) sets the effect of distance on migration success. In a recent mark-recapture study, 11 juvenile pikas were observed to migrate between patches (Peacock 1995; Peacock and Smith 1997). The shortest observed migration distance was $70 \mathrm{~m}$, the two longest distances were 210 and $396 \mathrm{~m}$, and the average migration distance for the remaining eight individuals was $98 \mathrm{~m}$ (Peacock 1995; Peacock and Smith 1997). Fit- ting the negative exponential distribution to these data gave $\alpha=5.28$. Unfortunately, this estimate is problematic because so few individuals were observed to migrate and especially because the mark-recapture study was conducted in only a part of the northern patch network, biasing the results toward short distances. We hence estimated $\alpha$ also using information in the observed patch occupancy patterns. To do this, we set $\alpha$ a free parameter and estimated it along with $x$ and $e^{\prime}$, using the four snapshot data sets using equations (1), (2), and (3b). This method gave an estimate of $0^{\prime}=2.34$. This estimate is substantially smaller than the one based on the limited mark-recapture data (5.28), but it is supported by some observed colonization events. Three colonization events were observed in the middle patch network (fig. 1) from 1989 to 1991, in the period when the southern part of the metapopulation went nearly extinct (Smith and Gilpin 1997). Two of these colonizations occurred more than $500 \mathrm{~m}$ from the nearest (few) occupied patches occupied in 1989. The most distant colonized patch was $650,1,000$, and $1,200 \mathrm{~m}$ away from the three closest occupied patches, which had one, four, and three pikas, respectively. To give an idea of the likelihood of such a colonization event, we calculated the probability of colonization in one time unit following the 1989 patch occupancy. For parameter values estimated with a low dispersal ability ( $\alpha=6.0$ ), this probability would be 0.00017 , whereas, for the parameters used in this study $(\alpha=2.5)$, the probability is 0.028 . Based on these considerations, we set the value of $\alpha$ at 2.5. We have performed simulations with other values of $\alpha$, and we found that the qualitative conclusions remain the same for $\alpha$, varying from 2 to 6 . We return to this point below.

After obtaining values for $\alpha, A_{0}$, and $b$, the remaining three parameters, $x, e$, and $y$, were obtained by minimizing equation (2) with the $J$ values calculated from equation (3b). We fitted the model separately to data from the four surveys as well as to the pooled data (table 1). Excepting the 1972 data, parameter estimation produced relatively consistent results, with the value of $x$ ranging from 1.04 to 1.36 and the value of $e^{\prime}$ from 0.0069 to 0.0174 . In 1972, patch occupancy was very high (fig. 1), hence there was little useful presence/absence information for reliable parameter estimation (for detailed maps of patch occupancy, see Smith and Gilpin 1997). Below we use parameter values estimated from the pooled data since, if available, it is preferable to use multiple snapshots of patch occupancy in parameter estimation (Hanski et al. 1996a; ter Braak et al. 1998).

\section{Model Simulation}

The dynamics of the pika metapopulation were investigated by simulating the incidence function model with 
Table 1: Parameter values of the incidence function model estimated using the four snapshots of patch occupancy available for the Bodie pika metapopulation

\begin{tabular}{lccccc}
\hline Data set & $\mathrm{x}$ & $\mathrm{e}^{\prime}$ & $\mathrm{y}$ & $\mathrm{e}$ \\
\hline 1972 & $2.12(.089)$ & $.0007(.0000)$ & 2.20 & .00014 \\
1977 & $1.36(.25)$ & $.0069(.0057)$ & 1.43 & .0034 \\
1989 & $1.23(.30)$ & $.013(.012)$ & 1.56 & .0058 \\
1991 & $1.04(.34)$ & $.017(.019)$ & 1.16 & .0130 \\
$1972-1991$ & $1.28(.29)$ & $.010(.094)$ & 1.50 & .0046 \\
\hline
\end{tabular}

Note: The last row gives the estimates using the pooled data; these latter values were used in the model simulations in this study. Asymptotic standard errors are given in parentheses. Values for $\alpha=2.5, A_{0}=0.015$, and $b=0.74$ were estinated with independent biological data as explained in the text. The systematic change in parameter values from 1972 to 1991 reflects the overall drop in patch occurancy during this period.

the parameter estimates obtained from the pooled data (table 1). Dynamics in the entire patch network were simulated, but the proportion of occupied patches, $p$, was recorded separately for the northern, middle, and southern parts of the network (fig. 1). Figure 2 summarizes the results.

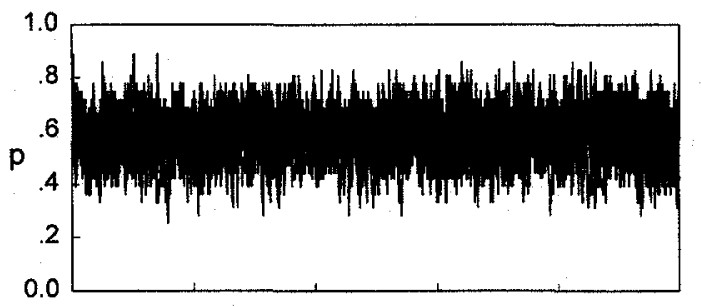

A
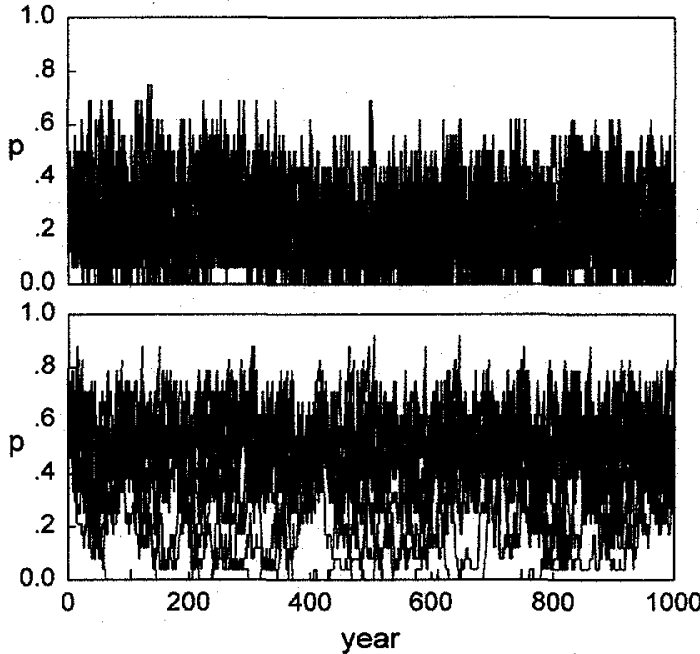

The metapopulation in the northern network was quasi-stable in the sense that the fraction of occupied patches fluctuated relatively little there and the metapopulation typically persisted for simulation runs of 1,000 time units (fig. $2 A, D$; we use "quasi-stable" to distinguish our meaning from the only mathematically sta-

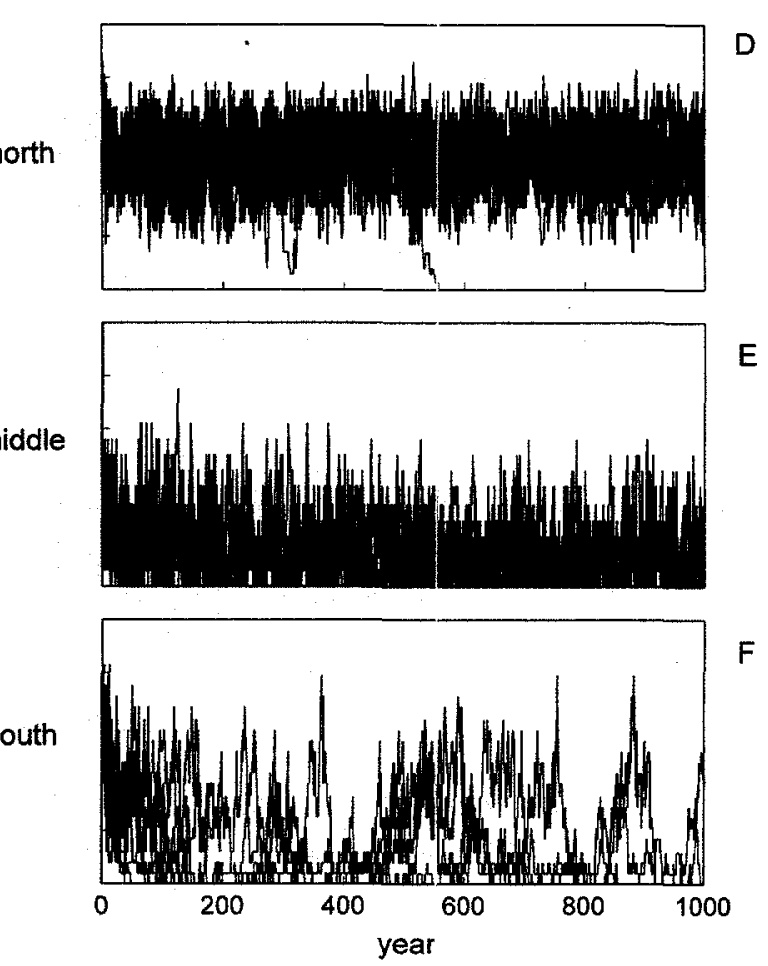

Figure 2: Patch occupancy in 10 replicate simulations of the Bodie pika metapopulation using the parameter values given in table 1. Each simulation was started by assuming the 1972 observed patch occupancy pattern and lasted for 1,000 time units. The metapopulation was iterated as a whole, but fluctuations in the proportion of occupied patches were recorded separately for the southern, middle, and northern parts of the network (fig. 1). $A-C$, Results for simulations with regional stochasticity set at $\sigma=0.1 ; D-$ $F$, results for $\sigma=0.2$. 

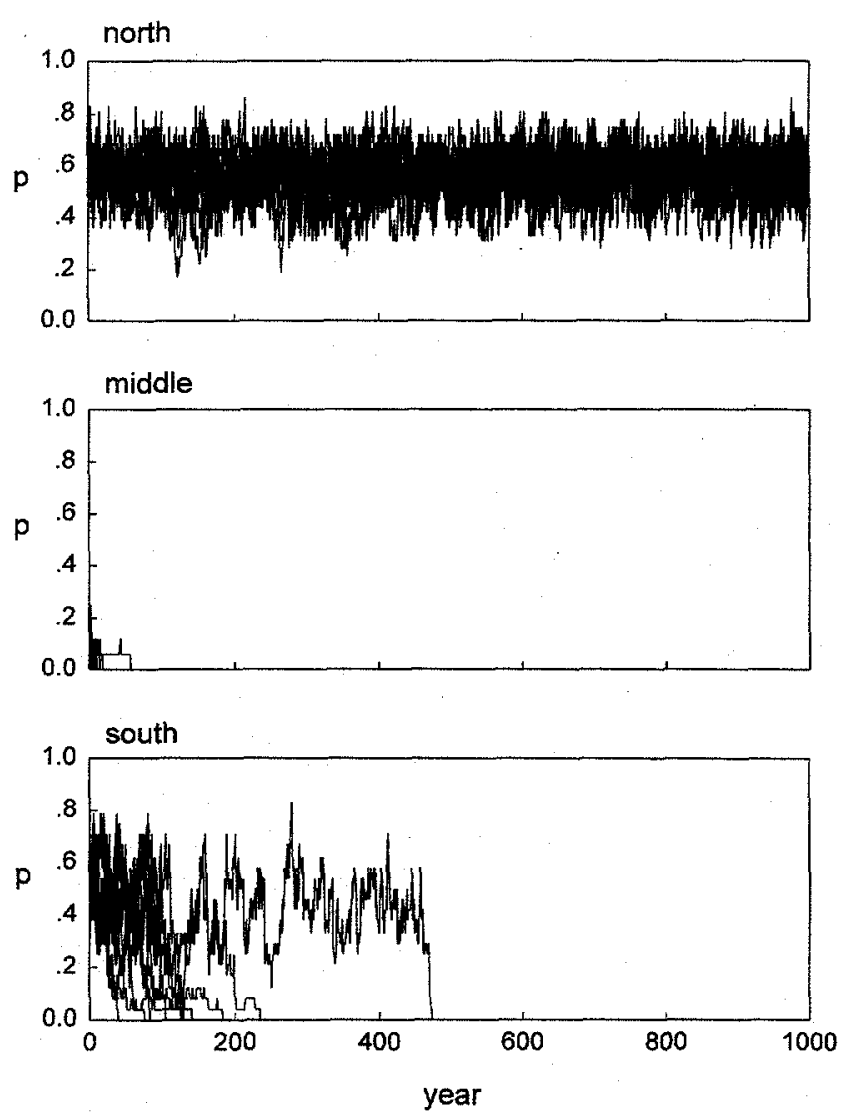

Figure 3: Patch occupancy in 10 replicate simulations with the northern, middle, and southern parts of the network simulated in isolation. These simulations included weak regional stochasticity, $\sigma=0.1$.

ble state in a stochastic metapopulation model, which is metapopulation extinction). The middle part of the network was very unstable, and the fraction of occupied patches frequently dropped to 0 (fig. $2 B, E$ ). Average patch occupancy in the southern network was higher than in the middle network, but patch occupancy is nonetheless predicted to drop often to 0 even without much regional stochasticity (fig. $2 \mathrm{C}$ ). With stronger regional stochasticity (fig. $2 F$ ), extinctions in the southern network are predicted to be increasingly frequent. The level of regional stochasticity assumed in figure $2 A-C$ was low. With a standard deviation of $\sigma=0.1$, the expected smallest ratio of modified patch area to the original area during 1,000 time units of simulation is $10^{(-2.88 \times 0.1)}=0.52$, where -2.88 is the 0.002 point of the cumulative normal distribution and, in a typical bad year, the multiplier for patch area is $10^{(-0.1)}=0.794$. In the subsequent analyses (except for fig. 4), we used this low level of regional stochasticity, $\sigma=0.1$.

Differences in the dynamic behavior of the three parts of the network become clearer when they are simulated
Table 2: Probabilities of observing steep metapopulation declines in the southern part of the network in simulation runs of $19 \mathrm{yr}$, starting from the pattern of patch occupancy observed in 1972

\begin{tabular}{lcc}
\hline & \multicolumn{2}{c}{ Probability of } \\
\cline { 2 - 3 } $\begin{array}{l}\text { Level of } \\
\text { stochasticity }\end{array}$ & Drop to $\mathrm{p} \leq .042$ & $\begin{array}{c}\text { Drop of } \geq .5 \\
\text { in } \mathrm{p}\end{array}$ \\
\hline$\sigma=.0$ & .000 & .042 \\
$\sigma=.1$ & .003 & .078 \\
$\sigma=.2$ & .047 & .302 \\
$\sigma=.3$ & .251 & .566 \\
\hline
\end{tabular}

Note: The observed decline in the southern network was from $p=$ .542 in 1972 to $p=.042$ in 1991 , which equals a drop of .5 in patch occupancy. This table reports the numbers of replicate simulations declining to $p=.042$ and the numbers of replicates experiencing a drop of .5 in $p$ between any two instances in simulation runs of $19 \mathrm{yr}$. The table is based on 1,000 replicate simulations. In similar simulations, the northern part of the metapopulation experienced steep declines infrequently and only when stochasticity was relatively high. For instance, with $\sigma=.3$, a drop to $p=.042$ was observed only eight times in 1,000 replicate simulations.

independently of each other (fig. 3). The northern network remains highly persistent by itself (fig. $3 A$ ), whereas the middle network is entirely unable to support a viable metapopulation (fig $3 B$ ). The behavior of the southern network was intermediate, but all 10 replicate simulations ended up in metapopulation extinction in $<500$ time units (fig. $3 C$ ).

The southern network exhibited a steep decline in patch occupancy from 0.54 in 1972 to 0.04 in 1991. We used the parameterized incidence function model to assess the probability of such a decline in $19 \mathrm{yr}$. With no regional stochasticity, the probability of observing a rapid decline is low, but this probability increases rapidly with an increasing level of regional stochasticity (table 2). In contrast, the northern patch network does not exhibit declines to low patch occupancy with $\sigma<0.25$ (fig. 2).

These results are not critically dependent on the parameter values used. The model was parameterized and run with different plausible combinations of $\alpha(2,4,6)$ and $A_{0}(0.01,0.015)$, the two parameters most difficult to estimate with the data available, and the results were similar to those reported in this article. As an example, figure 4 shows simulation results with parameter estimates based on $\alpha=6$ and $A_{0}=0.015$. No adequate data were available to estimate the value of $\sigma$, therefore we ran simulations with the value of $\sigma$ varying from 0.0 to 0.4 . The southern network occasionally lost the metapopulation even with $\sigma=0.0$ (not shown), whereas the northern network was persistent up to $\sigma>0.2$ with the standard parameter values (fig. 2D). In conclusion, the northern 

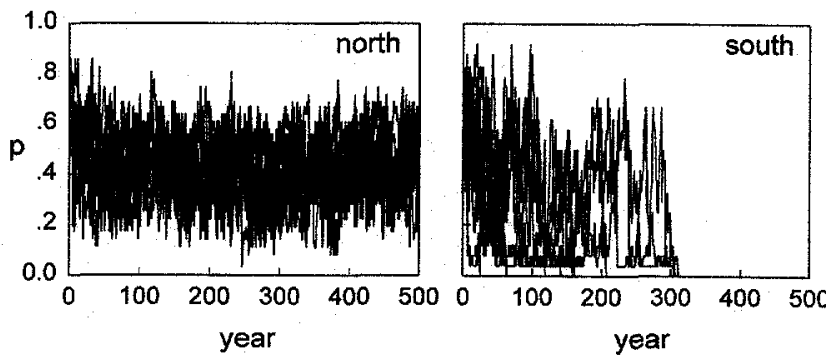

Figure 4: Patch occupancy in 10 replicate simulations using parameter values estimated with $\alpha=6$ and $A_{0}=0.015$. The entire patch network was simulated, and patch occupancy was recorded separately for north and south $(\sigma=0.3)$. The north survived in all replicates, whereas the south always went extinct. It is worth noting that the southern network was never successfully recolonized following extinction because of the short dispersal distances due to a large value of $\alpha$.

part of the metapopulation is much more persistent than the southern part regardless of the parameter combination used.

\section{Predicted Incidences and Population Turnover}

To investigate the model's ability to predict the occupancy in particular patches, we plotted the observed occupancy $(p)$ against the predicted incidence $(J)$, which was calculated from a simulation run of $32,000 \mathrm{yr}$ with $\sigma=0.1$ (fig. $5 A$ ). During this period, the metapopulation was sampled every $100 \mathrm{yr}$ at four distinct times spaced out as in the empirical censuses during years 1972-1991. Each quartet of data points generated one sample of predicted occupancy, and out of the 320 quartets, we calculated for each patch the predicted average occupancy and the associated $95 \%$ confidence intervals. Much of the scatter in figure $5 A$ is generated by the observed $p$ values being calculated from only four samples from a binomial distribution. Nonetheless, with two exceptions, the observed occupancy was within the 95\% confidence interval (fig. 5A). The Spearman rankcorrelation coefficient between the observed occupancy and the predicted incidence is $0.662, P<.00001$.

We next compared the model-predicted and observed sequences of patch occupancy patterns in the entire network. The idea here is to first construct a measure that characterizes the dynamic behavior of the fitted incidence function model. We then obtain the expected distribution of this measure by doing a large number of Monte Carlo simulations. Next we check whether the same measure calculated from the empirically observed sequence of patch occupancy patterns falls within the $95 \%$ confidence limits of predicted model behavior. If it does not, the model does not fit the data. To implement this test, we did the following. From the above simulation, we have
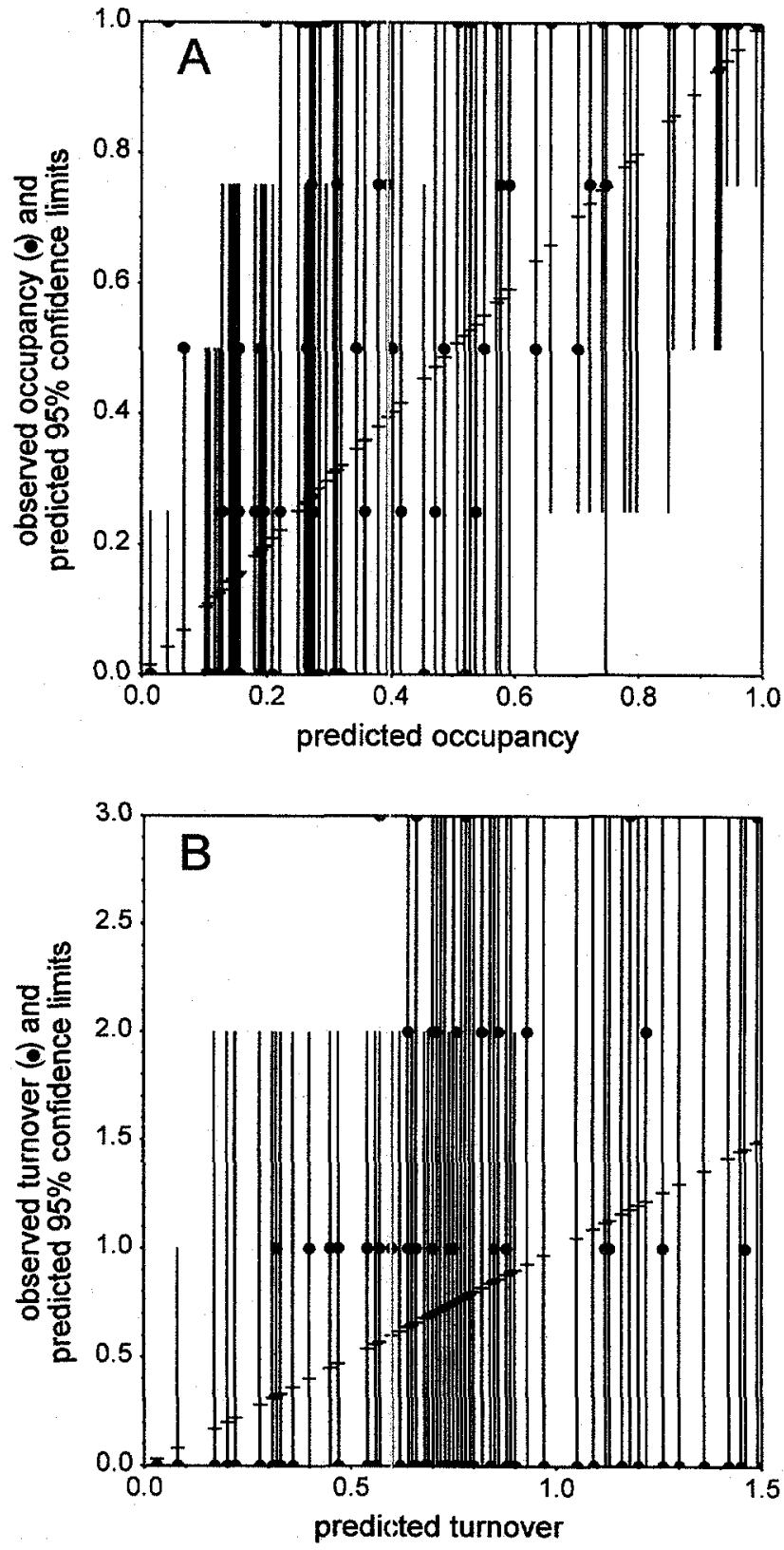

Figure 5: $A$, Predicted versus observed patch occupancies for all patches in the Bodie pika metapopulation. The patches have been arranged according to increasing predicted occupancy along the horizontal axis. The $95 \%$ confidence limits of the predicted occupancy are given by vertical lines. $B$, Similar analysis for predicted versus observed population turnover rate in individual patches. Note that, in the presence of the rescue effect, the predicted average long-tern turnover rate cannot exceed 0.5 times the maximum number of possible turnover events. 
Table 3: Observed and predicted (average \pm SD) numbers of turnover events in the pika metapopulation in the presence of regional stochasticity, $\sigma=.1$

\begin{tabular}{lccc}
\hline & \multicolumn{3}{c}{ Number of turnover events per interval (years) } \\
\cline { 2 - 4 } & $\begin{array}{c}1989-1991 \\
(2)\end{array}$ & $\begin{array}{c}1972-1977 \\
(5)\end{array}$ & $\begin{array}{c}1977-1989 \\
(12)\end{array}$ \\
\hline $\begin{array}{l}\text { Observed } \\
\text { Predicted }\end{array}$ & 14 & 18 & 17 \\
\hline
\end{tabular}

Note: The intervals are the time periods between the four complete censuses of the metapopulation.

320 quartets of occupancy patterns, all of which consist of 76 (sites) $\times 4$ (samples) $=304$ occupancy values of $J_{k}=0$ or $1(k=1, \ldots, 304)$. For each of the $304 J_{k}$ values, we calculated the mean occupancy, $m_{k}$. Next, for each of the 320 quartets, we calculated the sum over $k=$ $1-304$ of $\left(J_{k}-m_{k}\right)^{2}$, denoted by $W_{h}(h=1, \ldots, 320)$. For each of the 320 sets of four occupancy patterns, $W_{h}$ gives a value of variance from the mean $m_{k}$. If the $W$ value for the empirically observed set of patterns, $W_{0}$, is not within the $95 \%$ confidence interval of $W_{h}$, then the model is not supported. In our case $W_{\mathrm{o}}$ was at the $92.5 /$ 85 percentiles of $W_{h}$, when including/excluding the two outlier patches in the north. The fit of the model is, therefore, not rejected by this test. The two outlier patches, with low-predicted but high-observed occupancy, are due to the two northernmost patches that are somewhat isolated from the rest of the northern network (fig. 1) but close to a few middle-sized patches not censused because they are located on private land (Smith and Gilpin 1997). It is likely that presence of pikas on these uncensused patches explains the high-observed level of occupancy of these seemingly isolated patches.

The model was parameterized using only spatial data on patch occupancy. Another test of the model can therefore be made by comparing the predicted number of turnover events, extinctions, and colonizations with the observed one (table 3). For all three intervals between the four metapopulation censuses, the observed number of turnover events was within $1 \mathrm{SD}$ of that predicted by the model. There was thus a good match between the observed and predicted turnover rates. A patch-based analysis shows no significant difference between the observed and predicted turnover rates, but this analysis is not very informative because of the small number of possible turnover events (fig. $5 B$ ).

\section{Other Properties of the Patch Network}

The northern network has one large population with an estimated carrying capacity of 50 individuals, which might act as a minor mainland and, thus, strongly affect the dynamics of the metapopulation as a whole (Smith and Gilpin 1997). Using the estimated parameter values, this local population has, in the absence of regional stochasticity, an annual extinction risk of 0.003 . To analyze its role in the dynamics of the metapopulation, we repeated the simulations without this mainland patch. The northern patch network thereby became less stable: the average $p$ value was reduced, the amplitude of fluctuations in the $p$ value increased, and in most simulation runs of $1,000 \mathrm{yr}$, patch occupancy eventually dropped to 0 (fig. 6). However, even without the mainland patch, the northern network is more persistent than the southern one (fig. $3 C$ ). Thus, according to the model predictions, the observed difference between the northern and southern networks is only partly due to the presence of the largest patch in the northern network.

The role of the middle netivork (fig. 1) in the dynamics of the metapopulation is also worth consideration. By itself, the middle network with 16 small patches is very unstable (fig. $3 B$ ) and will not remain populated for more than a few years, a prediction that is consistent with empirical observations. Only $0.7 \%$ of the pooled colonization potential of the entire metapopulation is located in the middle patch network. However, it was evident from the simulation results that the middle network acted as a stepping-stone between the northern and the southern networks. We often observed that the southern and middle networks both went extinct and that the southern network was subsecuently recolonized via the middle network. The southern network is located so far from the northern one that direct colonization is unlikely, though not impossible, with the parameter values estimated from the data. This stepping-stone role of the middle network can be seen by examining the $p$ value distributions during model simulations. When the middle patch network was removed from the system, the southern part of the metapopulation spent significantly more time at extinction $(p=0)$ than when the middle patch network was present (fig. 7). Also, by comparing figures 2 and 3 , one notes that the presence of the northern network has a strong positive effect on patch occupancy in the middle and southern parts of the metapopulation.

Finally, to demonstrate the use of our measure of colonization potential with the pika metapopulation, we used equation (4) to rank the patches in an order of decreasing importance. We then removed the patch with the largest colonization potential and recalculated the colonization potentials for the remaining patches. We found that removing just the four most important patches (fig. 1) rendered the entire metapopulation highly unstable and prone to relatively rapid extinction (fig. 8 ). These four patches account for $73 \%$ of the pooled colonization 


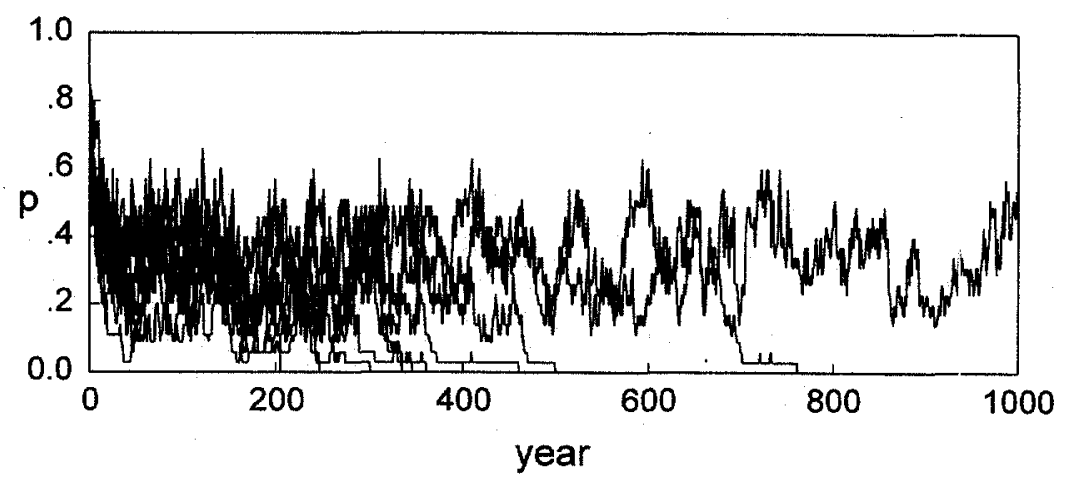

Figure 6: Patch occupancy in the northern network in 10 replicate simulations with the largest mainland patch (patch 1, fig. 1) removed $(\sigma=0.1)$.

potential in the metapopulation, whereas the 38 patches with the lowest colonization potentials accounted for $<1 \%$ of the pooled total.

\section{Discussion}

The incidence function model predicts that patch occupancy in the northern part of the patch network is quasistable, and the pika metapopulation is predicted to persist there for a long time, whereas the middle and southern parts of the network are predicted to show frequent steep declines in patch occupancy even in the absence of regional stochasticity. Our results suggest that the observed regional decline in patch occupancy in the southern part of the study area (fig. 1) can be explained by extinction-colonization dynamics in the presence of some regional stochasticity. In particular, these results demon- strate that no long-term environmental changes are needed to generate the observed decline. This is not to say that the observed pattern could not have other explanations, but our explanation is simple and plausible in the sense that it arises from the basic metapopulation dynamics, which are known to operate in this system. The model used in this study makes no biologically improbable assumptions, it was parameterized with empirical data, and it predicts well the population turnover rate and the occupancy of individual patches. We, therefore, conclude that the model gires a good description of the dynamics of this metapopulation.

The pika metapopulation has been previously studied with a two-dimensional stepping-stone model (Smith and Gilpin 1997). In this model, the southern part of the metapopulation was alway:s more persistent than the northern part, which is exactly the opposite to what we
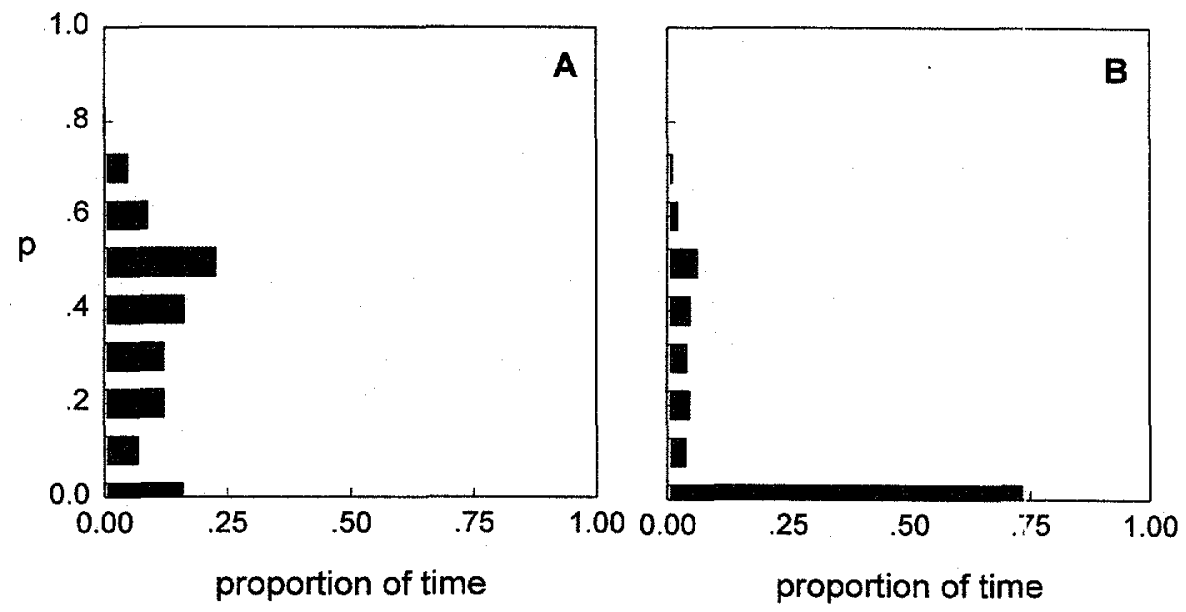

Figure 7: The distribution of the proportion of occupied patches in the southern patch network in model simulations $(\sigma=0.1)$. The results are given for simulations involving the entire patch network $(A)$ and for simulations with the middle part removed $(B)$. When the middle network was removed, the southern network remained extinct $(p=0)$ for $70 \%$ of the time, indicating that the unstable middle network is important in the recolonization of the southern network. The middle network thus operates as a functional stepping-stone. 


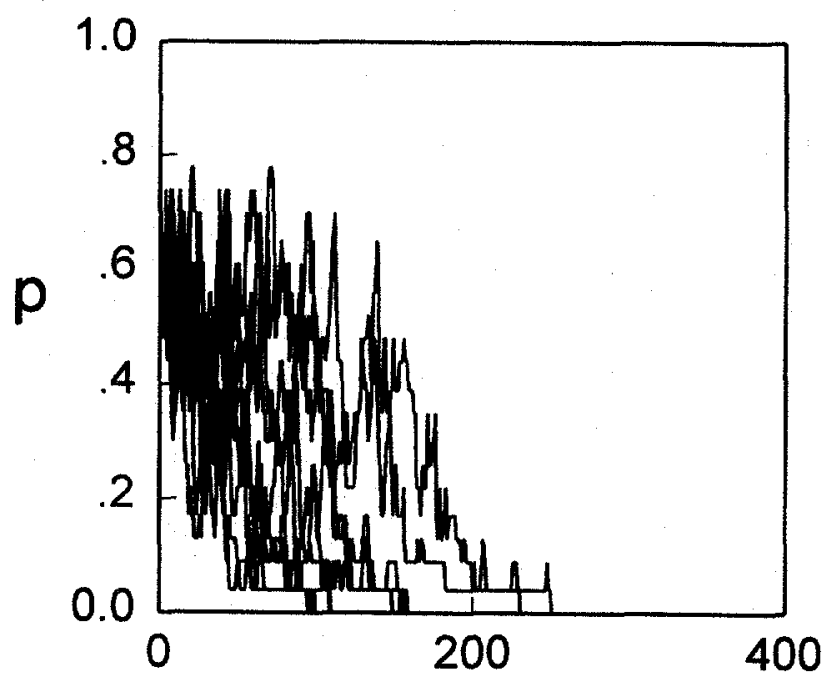

\section{year}

Figure 8: Simulation results $(\sigma=0.1)$ for the entire metapopulation excluding the four patches with the highest colonization potential (patches 1-4, fig. 1).

have found in this study and what has been found empirically. This striking conflict in model predictions is possibly caused by the unrealistic rectangular dispersal function used in the model presented earlier (Smith and Gilpin 1997). This function assumes that all patches within the dispersal radius are equally connected, whereas patches outside the radius are entirely unconnected. The greater stability in the south than in the north in the earlier predictions (Smith and Gilpin 1997) is possibly due to the average distance between patches being shorter in the south $(169 \mathrm{~m})$ than in the north $(204 \mathrm{~m}$ ). (We cannot conduct a more comprehensive analysis because the model in Smith and Gilpin 1997 was poorly documented.) The important message here is that a spatially explicit metapopulation model incorporating habitat patch sizes and positions may be inadequate for the study of particular metapopulations if the model assumptions are not otherwise realistic. Explicit habitat structure is not enough if the model is based on incorrect assumptions about the processes determining patch occupancy patterns, dispersal (determining colonization), and local dynamics (determining population extinction).

The incidence function model uses information in spatial patch occupancy patterns in parameter estimation. Another approach to modeling metapopulation dynamics uses information on observed population turnover events to independently parameterize functions for extinction and colonization probabilities (see, e.g., Sjögren Gulve and Ray 1996). These two approaches differ from each other in a fundamental way. The incidence function model assumes that the metapopulation is in a stochastic steady state, whereas models using turnover data in parameterization make no such assumption. In the latter approach, one easily obtains parameter estimates that predict an upward or downward trend in metapopulation size because, in the short time interval during which data typically are sampled, there is very likely to be an imbalance in the observed number of extinction and colonization events. In the data used in this study, there clearly is a strong declining trend in patch occupancy in the southern network. We have shown that this regional decline can be expected in this metapopulation due to extinction-colonization stochasticity only.

We have shown that the observed spatially correlated pattern in patch occupancy may arise from extinctioncolonization dynamics without any spatially correlated processes in local dynamics and population extinction. Our results strongly indicate that the long-term persistence of the Bodie pika metapopulation is dependent on the quasi-stable northern patch network. On the other hand, the results obtained with the measure of colonization potential (fig. 8) suggest that relatively few patches may ultimately be responsible for the persistence of the metapopulation. This is not entirely surprising since small or isolated patches will generally be of little importance to the dynamics of a metapopulation as a whole. However, without having a model to perform a quantitative analysis, it would have been difficult to assess the significance of individual patches to the long-term persistence of the metapopulation. Large patches naturally appear important since they have large and relatively persistent populations. However, assessing the importance of a patch for recolonization is very difficult without a quantitative model. The finding that the by-itself insignificant middle patch network is important as a steppingstone serves to remind us that even small and/or relatively isolated patches may tura out to be important for recolonization in metapopulations. This function of patches is not captured by the proposed measure of colonization potential, which basically measures localized interactions. What is ultimately needed for a comprehensive analysis of the system is the dynamic model itself.

In this study, we have used the incidence function model as a practical tool to assess the relative importance of individual patches to the long-term persistence of the metapopulation as well as to generate predictions about the performance of a species in particular patch networks. It is necessary to emphasize that, before making such predictions for management purposes, one must have justified well the use of the model for the particular system. We have done this for the Bodie pika metapopulation in this study (for two examples on butterfly meta- 
populations, see Hanski et al. 1996a; Wahlberg et al. 1996).

The theory of structured metapopulation dynamics (Hanski 1985; Gyllenberg and Hanski 1992; Hanski and Gyllenberg 1993; Hastings and Harrison 1994; Hanski et al. 1995b; Gyllenberg et al. 1997) predicts that metapopulations may have two alternative stable equilibria, one with most of the available habitat occupied and the other one corresponding to metapopulation extinction. In deterministic models, alternative equilibria arise from the presence of a strong rescue effect, which acts as a positive feedback mechanism in metapopulation dynamics. Regional stochasticity may cause the metapopulation to switch between the domains of the alternative stable equilibria. Assuming some migration from outside the network, which eliminates global metapopulation extinction as a stable equilibrium point, the hallmark of alternative stable equilibria is a bimodal distribution of the fraction of occupied patches during a long period of time (Hanski and Gyllenberg 1993; Hanski et al. 1995b). The southern network in the pika metapopulation shows a tendency toward a bimodal patch occupancy distribution (fig. 7), suggestive of alternative equilibria. However, the mechanism generating bimodality here is not exactly the same as in the above-cited models, which are deterministic and assume large networks. In real finite networks such as the one studied here, there are additional mechanisms that lead to bimodality and alternative quasi equilibria. The southern pika network is unlikely to stay for a long time with a small fraction of patches occupied. Either it goes extinct due to extinction-colonization stochasticity or many patches become occupied. Thus, in relatively small networks, the inevitable stochasticity in extinctions and colonizations amplifies the tendency toward bimodality due to the rescue effect in deterministic models.

Our results bear on questions about the structure and dynamics of populations that occur close to the range margin of the species, such as the Bodie pika metapopulation (Smith 1974a, 1974b). It has been commonly observed that average density decreases toward range margins (Smith 1974a; Hengeveld and Haeck 1982; Brown 1984; Hengeveld 1990; Maurer and Villard 1994). Recent studies of population variability have revealed that, additionally, variability typically increases toward the range margin (Thomas et al. 1994; Curnutt et al. 1996), where the population turnover rate is also highest. It is reasonable to assume that suitable habitat becomes increasingly sparse and more fragmented toward range margins (MacArthur 1972). In this case, our results predict decreased average abundance and increased amplitude of fluctuations in marginal populations. Decreasing density of suitable habitat may ultimately set the geographical range limit of a species (Carter and Prince 1981; Hanski 1991). The present results demonstrate that the location of the range boundary may shift without any systematic environmental changes, owing to extinction-colonization dynamics in sparse networks, especially if there is substantial regional stochasticity, as may be expected for marginal populations (Brown 1984; Curnutt et al. 1996).

\section{Acknowledgments}

We thank M. Camara, S. Harrison, T. Ives, J. Nagy, A. Taylor, C. Thomas, and an anonymous referee for several helpful comments on the manuscript.

\section{Literature Cited}

Brown, J. H. 1984. On the relationship between abundance and distribution of species. American Naturalist 124:225-279.

\section{Press, Chicago.}

Brown, J. H., and A. Kodric-Brown. 1977. Turnover rates in insular biogeography: effect of immigration on extinction. Ecology 58:445-449.

Carter, R. N., and S. D. Prince. 1981. Epidemic models used to explain biogeographical distribution limits. Nature (London) 293:644-645.

Curnutt, J. L., S. L. Pimm, ând B. A. Maurer. 1996. Population variability of sparrows in space and time. Oikos 76:131-144.

Gaston, H. J. 1994. Rarity. Chapman \& Hall, London.

Gyllenberg, M., and I. Hanski. 1992. Single-species metapopulation dynamics: a structured model. Theoretical Population Biology 42:35--61.

Gyllenberg, M., I. Hanski, and A. Hastings. 1997. Structured metapopulation models. Pages 93-122 in I. Hanski and M. Gilpin, eds. Metapopulation biology: ecology, genetics and evolution. Academic Press, London.

Hanski, I. 1985. Single-species spatial dynamics may contribute to long-term rarity and commonness. Ecology 66:335-343.

1991. Single-species metapopulation dynamics: concepts, models and observations. Pages 3-16 in $\mathrm{M}$. Gilpin and I. Hanski, edis. Metapopulation dynamics. Academic Press, London.

1994. A practical model of metapopulation dynamics. Journal of Animal Ecology 63:151-162.

- 1996. Metapopulation ecology. Pages 13-43 in D. E. Rhodes, Jr., R. K. Chesson, and M. H. Smith, eds. Population dynamics in ecological space and time. University of Chicago Press, Chicago.

1997. Predictive and practical metapopulation models: the incidence function approach. Pages 21-45 
in D. Tilman and P. Kareiva, eds. Spatial ecology. Princeton University Press, Princeton, N.J.

- 1998. Connecting parameters of local extinction and metapopulation dynamics. Oikos (in press).

Hanski, I., and M. Gyllenberg. 1993. Two general metapopulation models and the core-satellite hypothesis. American Naturalist 142:17-41.

Hanski, I., T. Pakkala, M. Kuussaari, and G. Lei. 1995a. Metapopulation persistence of an endangered butterfly in a fragmented landscape. Oikos 72:21-28.

Hanski, I., J. Pöyry, T. Pakkala, and M. Kuussaari. 19956. Multiple equilibria in metapopulation dynamics. Nature (London) 337:618-621.

Hanski, I., A. Moilanen, T. Pakkala, and M. Kuussaari. 1996a. Metapopulation persistence of an endangered butterfly: a test of the quantitative incidence function model. Conservation Biology 10:578-590.

Hanski, I., A. Moilanen, and M. Gyllenberg. 1996b. Minimum viable metapopulation size. American Naturalist 147:527-541.

Harrison, S. 1991. Local extinction in metapopulation context: an empirical evaluation. Pages 73-88 in $\mathrm{M}$. Gilpin and I. Hanski, eds. Metapopulation dynamics: empirical and theoretical investigations. Academic Press, London.

- 1994. Metapopulations and conservation. Pages 111-130 in P. J. Edwards, R. M. May, and N. R. Webb, eds. Large scale ecology and conservation biology. Blackwell, Oxford.

Hastings, A., and S. Harrison. 1994. Metapopulation dynamics and genetics. Annual Review of Ecology and Systematics 25:167-188.

Hastings, A., and K. Higgins. 1994. Persistence of transients in spatially structured models. Science (Washington, D.C.) 263:1133-1136.

Hengeveld, R. 1990. Dynamic biogeography. Cambridge University Press, Cambridge.

Hengeveld, R., and J. Haeck. 1982. The distribution of abundance. I. Measurements. Journal of Biogeography 9:303-316.

Levins, R. 1969. Some demographic and genetic consequences of environmental heterogeneity for biological control. Bulletin of the Entomological Society of America 15:237-240.

MacArthur, R. H. 1972. Geographical ecology. Harper \& Row, New York.

Maurer, B. A., and M. A. Villard. 1994. Geographic variation in abundance of North American birds. Research Exploration 10:306-317.

Peacock, M. M. 1995. Dispersal patterns, mating behavior, and population structure of pikas (Ochotona princeps). Ph.D. diss. Arizona State University, Tempe. Peacock, M. M., and A. T. Smith. 1997. The effect of habitat fragmentation on dispersal patterns, mating behavior, and genetic variation in a pika (Ochotona princeps) metapopulation. Oecologia (Berlin) 112:524533.

Pimm, S. L. 1991. The balance of nature. University of Chicago Press, Chicago.

Severaid, J. H. 1955. The natural history of the pikas (mammalian genus Ochotona). Ph.D. diss. University of California, Berkeley.

Sjögren Gulve, P., and C. Ray. 1996. Large scale forestry extirpates the pool frog: using logistic regression to model metapopulation dynamics. Pages 111-138 in D. R. McCullough, ed. Metapopulations and wildlife conservation and management. Island Press, Washington, D.C.

Smith, A. T. 1974a. The distribution and dispersal of pikas: consequences of insular population structure. Ecology 55:1112-1119.

- 1974b. The distribution and dispersal of pikas: influences of behavior and climate. Ecology 55:13861376.

1978. Comparative demography of pikas (Ochotona): effect of spatial and temporal age-specific mortality. Ecology 59:133-139.

- 1980. Temporal changes in insular populations of the pikas (Ochotona princeps). Ecology 60:8-13.

Smith, A. T., and M. Gilpin. 1997. Spatially correlated dynamics in a pika metapopulation. Pages 407-428 in I. Hanski and M. Gilpin, eds. Metapopulation biology: ecology, genetics and evclution. Academic Press, London.

Taylor, L. R., and I. P. Woiwod. 1980. Temporal stability as a density-dependent species characteristic. Journal of Animal Ecology 49:209-224.

ter Braak, C. J. F., I. Hanski, and J. Verboom. 1998. The incidence function approach to the modelling of metapopulation dynamics. Pages 167-188 in J. Bascompte and R. V. Solé, eds. Modelling spatiotemporal dynamics in ecology. Springer, Berlin.

Thomas, J. A., D. Moss, and E. Pollard. 1994. Increased fluctuations of butterfly populations towards the northern edges of species' range. Ecography 17:215220.

Wahlberg, N., A. Moilanen, and I. Hanski. 1996. Predicting the occurrence of species in fragmented landscapes. Science (Washington, D.C.) 273:1536-1538.

White, J. A., and R. F. Bowers. 1996. Host-pathogen systems in a spatially patchy environment. Proceedings of the Royal Society of London B, Biological Sciences 263:325-332.

Associate Editors: Anthony R. Ives Susan Harrison 